\title{
Feasibility of ultrasound-guided posterior approach for interscalene catheter placement during arthroscopic shoulder surgery
}

\author{
Hyeon Ju Shin, Jae Hyun Ahn, Hye In Jung, Choon Hak Lim, Hye Won Shin, Hye Won Lee, \\ Hae Ja Lim, Suk Min Yoon, and Seong Ho Chang \\ Department of Anesthesiology and Pain Medicine, College of Medicine, Korea University, Seoul, Korea
}

Background: Continuous interscalene block has been known to improve postoperative analgesia after arthroscopic shoulder surgery. This was a prospective study investigating the ultrasound-guided posterior approach for placement of an interscalene catheter, clinical efficacy and complications after placement of the catheter.

Methods: Forty-two patients undergoing elective arthroscopic shoulder surgery were included in this study and an interscalene catheter was inserted under the guidance of ultrasound with posterior approach. With the inplane approach, the $17 \mathrm{G}$ Tuohy needle was advanced until the tip was placed between the C5 and C6 nerve roots. After a bolus injection of $20 \mathrm{ml}$ of $0.2 \%$ ropivacaine, a catheter was threaded and secured. A continuous infusion of ropivacaine $0.2 \% 4 \mathrm{ml} / \mathrm{hr}$ with patient- controlled $5 \mathrm{ml}$ boluses every hour was used over 2 days. Difficulties in placement of the catheter, clinical efficacy of analgesia and complications were recorded. All patients were monitored for 48 hours and examined by the surgeon for complications within 2 weeks of hospital discharge.

Results: Easy placement of the catheter was achieved in $100 \%$ of the patients and the success rate of catheter placement during the $48 \mathrm{hr}$ period was $92.9 \%$. Postoperative analgesia was effective in $88.1 \%$ of the patients in the post anesthetic care unit. The major complications included nausea (7.1\%), vomiting (4.8\%), dyspnea (4.8\%) and unintended vascular punctures (2.4\%). Other complications such as neurologic deficits and local infection around the puncture site did not occur.

Conclusions: The ultrasound-guided interscalene block with a posterior approach is associated with a success high rate in placement of the interscalene catheter and a low rate of complications. However, the small sample size limits us to draw definite conclusions. Therefore, a well-designed randomized controlled trial is required to confirm our preliminary study. (Korean J Anesthesiol 2011; 61: 475-481)

Key Words: Complication, Continuous interscalene block, Posterior approach, Ultrasound.

Received: August 17, 2010. Revised: 1st, October 6, 2010; 2nd, January 12, 2011. Accepted: January 19, 2011.

Corresponding author: Hye Won Shin, M.D., Department of Anesthesiology and Pain Medicine, College of Medicine, Korea University, 126-1, Anam-dong 5ga, Seongbuk-gu, Seoul 136-705, Korea. Tel: 82-2-920-5632, Fax: 82-2-928-2275, E-mail: hwshin99@yahoo.com

(c) This is an open-access article distributed under the terms of the Creative Commons Attribution Non-Commercial License (http:// creativecommons.org/licenses/by-nc/3.0/), which permits unrestricted non-commercial use, distribution, and reproduction in any medium, provided the original work is properly cited. 


\section{Introduction}

Continuous interscalene block (CISB) has been known to improve postoperative analgesia after arthroscopic shoulder surgery [1]. By allowing real-time visualization of needle advancement, ultrasound-guided nerve block makes it possible to avoid direct contact with and injection of local anesthetic into the target nerve [2] and helps to place the catheter in the appropriate location within the brachial plexus during shoulder surgery involving the $\mathrm{C} 5$ and $\mathrm{C} 6$ nerve roots $[3,4]$.

Antonakakis et al. [4] described that the ultrasound-guided posterior approach had the advantage of securing catheter placement because it passed through multiple muscle layers. When the interscalene brachial plexus block was performed using traditional techniques [5], spread of local anesthetic to the phrenic nerve through the anterior scalene fascia has been reported, with an incidence rate as high as $100 \%$ [6]. However, by using a lower dose of local anesthetic with the help of ultrasound-guidance, the incidence rate of complications such as hemidiaphragmatic paresis can be reduced $[7,8]$. A study that showed CISB using ultrasound-guided posterior approach was more effective than a single injection interscalene block [9]. But, there are few studies that examined the success rate of catheter placement and complications such as respiratory distress and nerve injury.

We evaluated the success rate of catheter placement and the effectiveness of pain control by CISB using ultrasoundguided posterior approach as well the incidence of nausea, vomiting, dyspnea, vascular puncture, infection and neurologic complications.

\section{Materials and Methods}

The subjects examined in this study were 42 American Society of Anesthesiologists (ASA) physical status I-II in-patients undergoing elective arthroscopic rotator cuff repair. The Institutional Review Board of the Hospital approved this study and written informed consent was obtained from each patient. Patients who received chronic analgesic therapy, had severe chronic bronchopulmonary disease, coagulopathy, neuropathy or allergy to amide local anesthetic were excluded.

Upon the patient's arrival in the operative room, noninvasive monitoring of blood pressure, EKG and pulse oximetry were performed and 1-2 mg of midazolam was given intravenously to the patient as premedication.

An experienced anesthesiologist performed the ultrasoundguided CISB and the surgery was done by one operator. CISB was performed using the ultrasound-guided posterior approach described by Antonakakis et al. [4] and an interscalene catheter placement method as previously reported [10]. The patient was placed in the lateral decubitus position with the operative shoulder nondependent. A linear high frequency 6-13 MHz ultrasound probe (Sonosite M-Turbo ${ }^{\circledR}$, Sonosite Bothell, USA) was placed on supraclavicular fossa and the brachial plexus was identified. On short axis view, the subclavian artery was identified and the brachial plexus was seen superficial and posterior to the artery. While maintaining the brachial plexus in the center of the image, the probe was moved cephalad direction until the brachial plexus was located between the anterior and middle scalene muscles at the $\mathrm{C} 7$ vertebra level. At this point, a povidone iodide skin preparation was used, and an ultrasound transducer was prepared with a sterile vinyl wrap (Tegaderm $^{\mathrm{TM}}$, 3M, Germany) and sterile gel (Progel ${ }^{\circledR}$, Dayo, Korea). The subcutaneous tissue was then anesthetized with $3 \mathrm{ml}$ of $2 \%$ lidocaine (Lidocaine ${ }^{\circledR}$, Huons, Korea). A 17-gauge Tuohy needle (Arrow ${ }^{\circledR}$, Arrow International, USA) was inserted between the levator scapulae and trapezius muscles (Fig. 1). The advancement was done with the in-plane needle insertion technique to clearly visualize the needle. The needle was advanced until it was located between the C5 and C6 nerve roots of the brachial plexus. After the insertion of a 17-gauge Tuohy needle into the anterior border of the middle scalene muscle, $20 \mathrm{ml}$ of $0.2 \%$ ropivacaine (Naropin ${ }^{\circledR}$, Astrazeneca, UK) was injected into the interscalene space to expand the fascial plane between the brachial plexus and middle interscalene muscle (Fig. 2). After a 19-gauge catheter was advanced through the 17-gauge Tuohy needle, $10 \mathrm{ml}$ of normal saline was injected through the catheter and expansion of the interscalene space was confirmed by using ultrasound (Fig. 3). When the resistance to the catheter threading was noted, the catheter was advanced after turning the Tuohy needle bevel to the caudad direction. After the 17-gauge Tuohy needle was removed from the

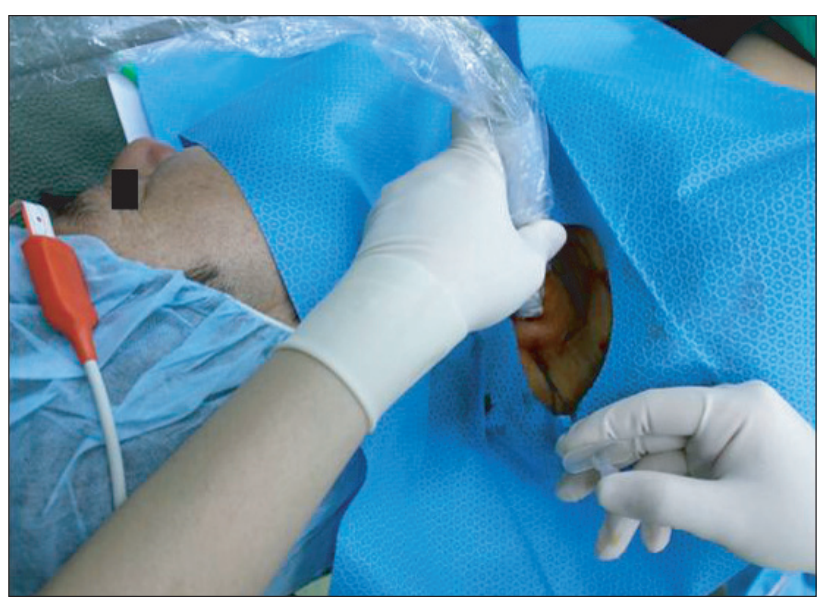

Fig. 1. Patient positioning and transducer location to develop a short axis view of the brachial plexus at the level of the cervical root. The Tuohy needle was inserted using the in plane insertion technique. 


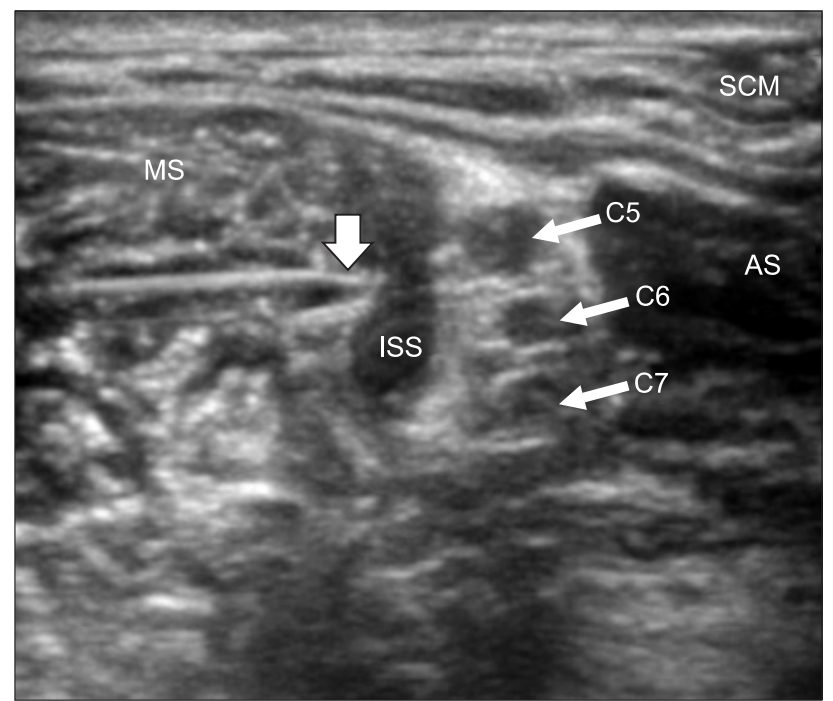

Fig. 2. Ultrasound image of the interscalene area after the injection of local anesthetic. The bevel of the Tuohy needle (arrow) was visualized between the $\mathrm{C} 5$ and $\mathrm{C} 6$ nerve roots. The interscalene space had been expanded with $20 \mathrm{ml}$ of local anesthetic. AS: anterior scalene muscle, MS: middle scalene muscle, SCM: sternocleidomastoid muscle, ISS: interscalene space.

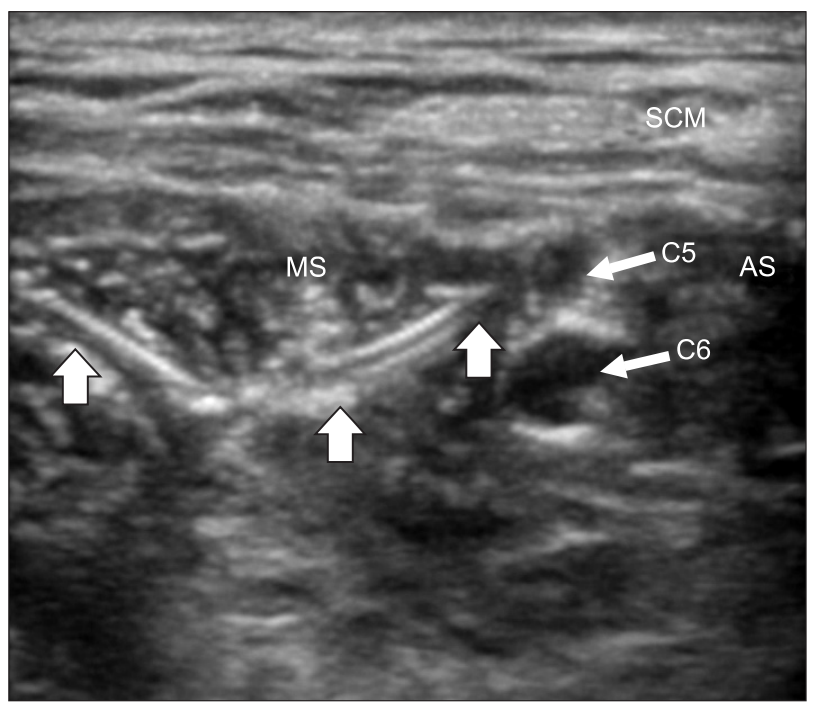

Fig. 3. After the Tuohy needle was removed, the catheter was clearly visualized as it was embedded in the muscles of the neck. The spreading of local anesthetic was through the end holes of the catheter between the C5 and C6 nerve roots. The large arrows outline the catheter. AS: anterior scalene muscle, MS: middle scalene muscle, SCM: sternocleidomastoid muscle.

catheter, the catheter was subsequently secured to the skin with 2-0 black silk preventing removal of the catheter (Fig. 4). While performing CISB, frequent aspiration using a $10 \mathrm{ml}$ syringe was performed to check for blood and CSF due to vascular puncture, accidental spinal or epidural anesthesia. Then, the

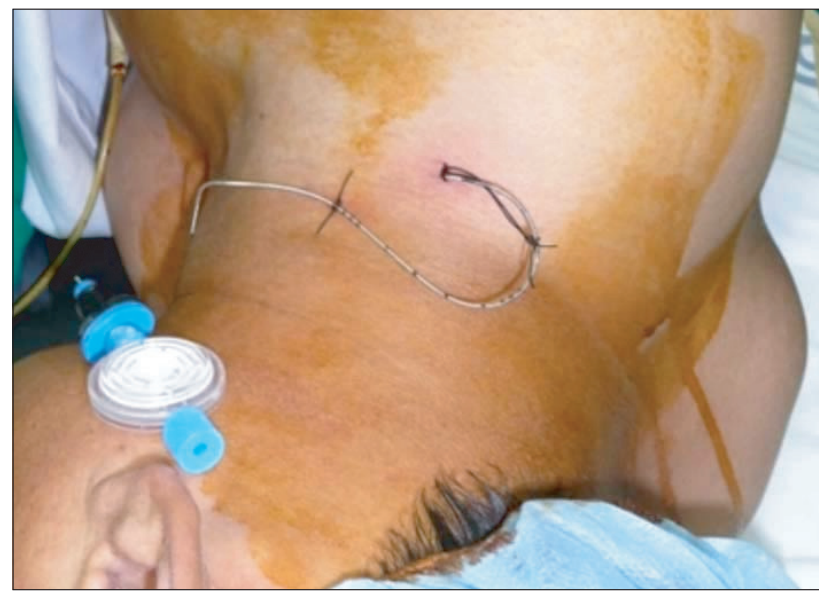

Fig. 4. The catheter was sutured on the skin after placement by black silk for continuous interscalene block.

distance from the skin to the tip of the catheter and from the Tuohy needle tip to the tip of the catheter was recorded. Ten minutes after the procedure, the brachial plexus block was evaluated using an alcohol swab and was considered to be successful when subjects demonstrated a decrease in perceived sensation to cold on the skin over the deltoid muscle. Also, the success rate of catheter placement after a single attempt was evaluated.

General anesthesia was induced by IV administration of $2 \%$ lidocaine $(1 \mathrm{ml})$ and propofol $(2 \mathrm{mg} / \mathrm{kg})$ after manual ventilation was performed with $\mathrm{O}_{2}$ given at $8 \mathrm{~L} / \mathrm{min}$. At loss of eyelash reflex, rocuronium $(0.6 \mathrm{mg} / \mathrm{kg})$ was administered intravenously and intubation was performed. Anesthesia was maintained by $\mathrm{O}_{2}$ and $\mathrm{N}_{2} \mathrm{O}$ each at $2 \mathrm{~L} / \mathrm{min}$, sevoflurane was administered at $1.5-2.0 \mathrm{vol} \%$. After the surgery, pyridostigmine (10 mg) and glycopyrrolate $(0.4 \mathrm{mg}$ ) were intravenously administered and the patient was extubated. Before transferring the patient to the post anesthetic care unit (PACU), a patientcontrolled analgesia (PCA) pump (Accumate ${ }^{\circledR} 1000$, Wooyoung Medical, Korea) containing $280 \mathrm{ml}$ of $0.2 \%$ ropivacaine, with a continuous basal infusion rate at $4 \mathrm{ml} / \mathrm{hr}$ and a patientcontrolled boluses available $5 \mathrm{ml}$ every hour, was connected to the patient. The catheter was removed at 48 hours after completion of the surgery. Clinical effectiveness of pain control in the PACU was evaluated. Fentanyl $(25-100 \mu \mathrm{g})$ was administered to the patient for severe pain and when needed ketorolac (30 mg) was given additionally. When the patient had continued pain, $10 \mathrm{ml}$ of $1 \%$ lidocaine was injected through the catheter. For patients with nausea or vomiting, metoclopramide (10 mg) or ondansetron (8 mg) was administered. Success rate of the catheter placement was evaluated for the next 48 hours at the ward. Patients were closely monitored for signs of dyspnea immediately after catheter placement and also during 
Table 1. Demographic Data

\begin{tabular}{lc}
\hline & $\mathrm{n}=42$ \\
\hline Age (yr) & $56.4 \pm 11.2$ \\
Weight (kg) & $64.5 \pm 8.4$ \\
Height (cm) & $161.4 \pm 9.1$ \\
Sex (M/F) & $20 / 22$ \\
ASA physical status (I/II) & $17 / 25$ \\
Operation time (min) & $86.5 \pm 31.8$ \\
\hline
\end{tabular}

Values are mean \pm SD or number of patients.

Table 2. Block Outcome after Placement of the Continuous Interscalene Catheter

\begin{tabular}{lc}
\hline & $\mathrm{n}=42$ \\
\hline Catheter length through Tuohy needle tip (cm) & $5.4 \pm 1.8$ \\
Catheter length from skin (cm) & $9.6 \pm 2.2$ \\
Success rate of sensory block on deltoid muscle (\%) & 100 \\
Easy placement of the catheter (one attempt) (\%) & 100 \\
Success rate of catheter placement during 48 hrs (\%) & 92.9 \\
\hline
\end{tabular}

Values are mean \pm SD

their stays at the PACU and the ward. Incidence of infection was evaluated using the following criteria - fever, itching sensation and pain at site of catheter placement, redness of skin and abscess formation at site of catheter placement during dressing at postoperative day-1, postoperative day-2, and catheter removal before patient was discharged. Incidence of neurologic complications, such as loss of sensation, paresthesia, hyperesthesia, hypoesthesia, weakness of muscle power and paralysis unrelated to the surgical procedure was evaluated during the patient's hospital stay and two weeks after discharge during outpatient visit.

All measured data were expressed as mean \pm SD, number of patients, or percentage (\%).

\section{Results}

Among the forty-two patients included in this study, twenty were male and twenty-two were female. Age, body weight, height, sex, ASA class and operation time of patients are shown in Table 1.

The needle was originally positioned in the interscalene space of C5 and C6 nerve roots and the catheter was threaded $5.4 \pm 1.8 \mathrm{~cm}$ further from the tip of the Tuohy needle and finally positioned at a depth of $9.6 \pm 2.2 \mathrm{~cm}$ from the skin. Initial catheter placement was successful in all patients, but due to accidental removal of the catheter in the ward in three of the patients, the success rate of catheter placement during the 48 hours was $92.9 \%$. Ten minutes after injection of local anesthetic into the interscalene space, a decrease in cold sensory function of the ipsilateral deltoid muscle was observed in all patients
Table 3. Analgesic Requirement and Complications after Placement of the Continuous Interscalene Catheter

\begin{tabular}{lc}
\hline & $\mathrm{n}=42$ \\
\hline $\begin{array}{l}\text { Patients requiring analgesics in PACU } \\
\text { Patients with complications }\end{array}$ & $5(11.9)$ \\
$\quad$ (during interscalene catheter placement) & \\
$\quad$ Unintended vascular punctures & $1(2.4)$ \\
$\quad$ Inadvertent spinal anesthesia & 0 \\
$\quad$ Inadvertent epidural anesthesia & 0 \\
(after 48 hrs) & $3(7.1)$ \\
$\quad$ Nausea & $2(4.8)$ \\
Vomiting & $2(4.8)$ \\
Dyspnea & \\
(after 14 days) & 0 \\
Local infection around the puncture site & 0 \\
$\quad$ Neurological deficits & \\
\hline
\end{tabular}

Values are number (\%) of patients. PACU: post-anesthetic care unit.

(100\%) (Table 2). Five patients (11.9\%) needed analgesics in the PACU and they were given 25-100 $\mu$ g of fentanyl. Three of the five patients were additionally injected with $30 \mathrm{mg}$ of ketorolac, and the remaining two with more severe pain were injected with $10 \mathrm{ml}$ of $1 \%$ lidocaine via the catheter. During the procedure, hematoma, although minor, occurred in 1 patient (2.4\%) due to puncture of a small vessel. However, there were no cases of inadvertent spinal and epidural anesthesia. Five patients (11.9\%) complained of nausea and vomiting and were administered either metoclopramide (10 mg) or ondansetron (8 $\mathrm{mg}$ ). Two patients (4.8\%) complained of dyspnea with anxiety, but breathing soon normalized when the PCA pump was locked.

During infusion of local anesthetic, 19 patients (45.2\%) experienced paresthesia or numbness of the arm, but sensory and motor function returned to normal in all patients after transient discontinuation of local anesthetic infusion or after removal of the catheter. No patient showed signs of infection around the area of the catheter placement or abnormal sensory as well as lack of motor nerve function of the shoulder, arm and hand during admission and two weeks after discharge (Table 3).

\section{Discussion}

CISB is an effect analgesic method for shoulder surgery [1]. By using ultrasound to visualize the nerves, nearby anatomical structures and spread of local anesthetic, it is possible to perform the nerve block with a high success rate without causing unintended nerve stimulation or paresthesia [2].

The posterior approach to the brachial plexus at the level of the cervical nerve roots was first described by Kappis in 1912 [11] and ultrasound-guided posterior approach for the placement of the interscalene catheter was subsequently reintroduced by Antonakakis et al. in 2009 [4].

Challenges related to the anterolateral approach of CISB 
include avoiding the external jugular vein, catheter dislodgement and inclusion of the catheter site in the surgical field. Furthermore, failure rate of up to $20 \%$ was reported while using the anterolateral approach with a nerve stimulator [12,13]. Reported failure rates of initial catheter placement were estimated to be $8 \%$ [14]. On the other hand, as the ultrasound-guided posterior approach passes many muscles, the catheter can be safely positioned [4]. When the catheter is placed on the neck, as the distance between skin and the brachial plexus becomes short, there is a risk of the catheter being inadvertently removed from the originally positioned site. Therefore, to improve the success rate of the catheterization, the catheter should be positioned to the proper depth and sutured to the skin. In this study, the catheter was inserted between the levator scapular and trapezius muscles with a depth of $9.6 \pm 2.2 \mathrm{~cm}$ from the skin, which was $5.4 \pm 1.8 \mathrm{~cm}$ from the needle tip positioned between the C5 and C6 nerve roots. The three patients who experienced inadvertent removal of the catheter had their catheter positioned at a depth no longer than $7 \mathrm{~cm}$ from the skin and $3 \mathrm{~cm}$ from the $\mathrm{C} 5$ and $\mathrm{C} 6$ nerve roots. However, when the catheter is positioned deeply, kinking or knoting can occur and complications such as intrathecal or epidural insertion can happen. Therefore, the catheter should be positioned at an appropriate depth that can prevent inadvertent catheter removal.

The acute complications with CISB are transient phrenic nerve palsy, recurrent laryngeal nerve paralysis, Horner's syndrome, pneumothorax, hemothorax, spinal anesthesia and epidural anesthesia. The phrenic nerve usually starts at the C4 nerve root but also starts from C3 and C5 nerve roots. The phrenic nerve than travels obliquely with the internal jugular vein and passes the anterior border of the anterior scalene muscle. The group of interscalene blocks by the Winnie technique [5] has high rate of pherenic nerve palsy [6]. In a study with cadavers using the supraomohyoidal block, an approach that avoids medially directed needle advancement, the solution did not spread medially beyond the lateral margin of the anterior scalene muscle into the scalenovertebral triangle. Therefore, the phrenic nerve, stellate ganglion, laryngeal nerve and the vertebral artery were not exposed to the injected solution. Whereas in the cadavers group received an interscalene block by the Winnie technique, the dye spreaded medially to the anterior scalene muscle and reached the phrenic nerve, the vagus nerve and the sympathetic trunk [15]. Urmey et al. [6] showed that $100 \%$ of the patients with an interscalene brachial plexus anesthesia by the Winnie technique developed ipsilateral hemidiaphragmatic paresis detected by ultrasonography. Ultrasound-diagnosed phrenic nerve palsy was reported to be detected in $85 \%$ of the brachial plexus block without nerve stimulator, $35 \%$ with nerve stimulator and
$20 \%$ in CISB patients [16]. In CISB with nerve stimulator and the modified lateral technique, $15 \%$ of the patients showed dyspnea due to phrenic nerve palsy. They were usually elder patients and recovered in about 4 hours with oxygen therapy [17]. In another case report, a patient who had undergone CISB complained of chest pain the next day. In the chest x-ray, the ipsilateral diaphragm was elevated due to unilateral phrenic nerve block, and atelectasis and the pleural effusion was also detected [18]. However, within ultrasound guidance and low dose local anesthetic, the incidence rate of unilateral phrenic nerve block can be decreased [7,8]. Renes et al. [7] performed a brachial plexus block with $10 \mathrm{ml}$ of local anesthetic and the incidence rate of unilateral phrenic nerve palsy was $93 \%$ with nerve stimulator and $13 \%$ with ultrasound-guidance. Riazi et al. [8] performed an interscalene brachial plexus block under the ultrasound-guidance and reported the incidence rate of unilateral phrenic nerve palsy to be $100 \%$ with $20 \mathrm{ml}$ of local anesthetic and $45 \%$ with $5 \mathrm{ml}$ but there was no statistically significant difference in pain scores for 24 hours. In our study, $20 \mathrm{ml}$ of local anesthetic was injected to the interscalene space and $4.8 \%$ of patients complained of dyspnea with anxiety. In those cases, PCA was locked and calmed the patient and the respiration was restored. Compared to other studies, the number of patients complaining dyspnea was rather small. First, this was due to the fact that almost all of the patients were confirmed by ultrasound to have the local anesthetic not crossing the anterior scalene muscle to the medial side and being well spread to the space formed between the brachial plexus and the middle scalene muscle (Fig. 2). Second, the catheter was confirmed not to be crossing the scalene muscle to the medial side and well positioned in the space formed between the brachial plexus and the middle scalene muscle (Fig. 3). Third, with injection of normal saline into the catheter, the spread of saline in the space between the C5 and C6 nerve roots and middle scalene muscle was also confirmed by ultrasound. However, it is difficult to confirm the catheter positioning by ultrasound in all patients. A lower dose of local anesthetic will further decrease the incidence rate of unilateral phrenic nerve palsy.

A tingling sensation maintained for hours after CISB can cause anxiety and discomfort to patients. In this study, $45.2 \%$ of patients complained of paresthesia. However, after discontinuing the drug or removal of the catheter, every patient had their sensory and motor function normalized. This can be explained with the accumulation effect with the initial loading dose before general anesthesia and continuous infusion dose after the surgery. In a report of Fredrickson and Kilfoyle [19] new neurologic symptoms appeared after 10 days, $8.2 \%, 3.7 \%$ in a month and $0.6 \%$ in 6 months of a thousand patients who had orthopedic surgery under ultrasound-guided peripheral nerve 
block. Almost all of the neurologic symptoms were not related to the nerve block. New neurologic symptoms appeared more often in patients who experienced paresthesia during the procedure and the incidence rate was similar to the traditional procedure. Antonakakis et al. [4] with the posterior approach inserted a needle between the C5 and C6 nerve roots and punctured the sheath of the brachial plexus and injected the local anesthetic and threaded the catheter. As this method can have the possibility of nerve damage due to the proximity to the nerve, we placed the needle into the interscalene space but did not go closely near the C5 and C6 nerve roots to puncture the sheath of the brachial plexus nerve (Fig. 2). No patient complained of the severe paresthesia due to the needle contacting the nerve nor patient complained of sensory and motor nerve abnormalities 2 weeks after surgery at the orthopedic outpatient department.

During the CISB, one should avoid puncture of the vertebral artery. As the vertebral artery starts from the subclavian artery, passes between the longus colli and anterior scalene muscles, and goes into the transverse foramen of the C6 vertebra, it can be confirmed at the level of the $\mathrm{C} 7$ vertebra with the ultrasound. With the posterior approach with ultrasound, since the needle is not inserted into the anterior scalene muscle, one can avoid puncture of vertebral artery. In this study, there were no arterial punctures including the vertebral artery. One of the patients had a venous puncture and a small hematoma, but it disappeared at the end of the surgery.

Infection of the CISB catheter can cause significant complications. The risk factors for the infection of the catheter include admission to the intensive care unit, immunocompromised patients, catheter insertion site and duration of catheter placement [20]. Capdevila et al. [21] reported a case with acute neck cellulitis and mediastinitis caused from an infection related to the process of local anesthetic injected into the PCA. In many studies, 48 hours after the peripheral nerve block, $16.7-57 \%$ of patients had bacterial infection, but only $3.0-9.0 \%$ had signs of the local infection, and the interscalene catheter had bacterial infection in $11.0-25 \%$ of the cases [22,23]. In our study during the admission period and the outpatient department follow-up 2 weeks later, there were no signs of infection around the procedure site of the neck.

This study has potential limitations. First, as the interscalene space is not a closed area, local anesthetic can spread slowly to the anterior scalene muscle or spread rostrally and make phrenic nerve palsy, even if this did not occur initially. Therefore, to record the precise incidence rate of phrenic nerve palsy, ultrasound and a chest X-ray would be needed. Second, to confirm the location of the catheter, agitated solution or color doppler will be needed. Third, in this study we recorded the complication rate according to the catheter insertion technique. It will be helpful to record the pain scores of the patients during
48 hours to confirm the usefulness in this catheter placement technique.

In conclusion, ultrasound-guided posterior approach for interscalene catheter placement for arthroscopic shoulder surgery showed high success rate and good pain control with low complication rates such as dyspnea, inadvertent puncture of the vessel, infection and neurologic abnormalities. However, for a safer procedure, further study is needed about the method of higher success rate and lower complication rate including larger scale patient group.

\section{References}

1. Fredrickson MJ, Ball CM, Dalgleish AJ. Analgesic effectiveness of a continuous versus single-injection interscalene block for minor arthroscopic shoulder surgery. Reg Anesth Pain Med 2010; 35: 2833.

2. Marhofer P, Chan VW. Ultrasound-guided regional anesthesia: current concepts and future trends. Anesth Analg 2007; 104: 1265-9.

3. Fredrickson MJ, Ball CM, Dalgleish AJ. A prospective randomized comparison of ultrasound guidance versus neurostimulation for interscalene catheter placement. Reg Anesth Pain Med 2009; 34: 590-4.

4. Antonakakis JG, Sites BD, Shiffrin J. Ultrasound-guided posterior approach for the placement of a continuous interscalene catheter. Reg Anesth Pain Med 2009; 34: 64-8.

5. Winnie AP. Interscalene brachial plexus block. Anesth Analg 1970; 49: 455-66.

6. Urmey WF, Talts KH, Sharrock NE. One hundred percent incidence of hemi diaphragmatic paresis associated with interscalene brachial plexus anesthesia as diagnosed by ultrasonography. Anesth Analg 1991; 72: 498-503.

7. Renes SH, Rettig HC, Gielen MJ, Wilder-Smith OH, van Geffen GJ. Ultrasound-guided low-dose interscalene brachial plexus block reduces the incidence of hemidiaphragmatic paresis. Reg Anesth Pain Med 2009; 34: 498-502.

8. Riazi S, Carmichael N, Awad I, Holtby RM, McCartney CJ. Effect of local anaesthetic volume ( $20 \mathrm{vs} 5 \mathrm{ml}$ ) on the efficacy and respiratory consequences of ultrasound-guided interscalene brachial plexus block. Br J Anaesth 2008; 101: 549-56.

9. Mariano ER, Afra R, Loland VJ, Sandhu NS, Bellars RH, Bishop $\mathrm{ML}$, et al. Continuous interscalene brachial plexus block via an ultrasound-guided posterior approach: a randomized, triplemasked, placebo-controlled study. Anesth Analg 2009; 108: 168894.

10. Swenson JD, Davis JJ, DeCou JA. A novel approach for assessing catheter position after ultrasound-guided placement of continuous interscalene block. Anesth Analg 2008; 106: 1015-6.

11. Kappis M. Conduction anesthesia of abdomen, breast, arm, and neck with paravertebral injection (German). Munchen Med Wochenschr 1912; 59: 794-6.

12. Ilfeld BM, Morey TE, Wright TW, Chidgey LK, Enneking FK. Continuous interscalene brachial plexus block for postoperative pain control at home: a randomized, double-blinded, placebocontrolled study. Anesth Analg 2003; 96: 1089-95. 
13. Grant SA, Nielsen KC, Greengrass RA, Steele SM, Klein SM. Continuous peripheral nerve block for ambulatory surgery. Reg Anesth Pain Med 2001; 26: 209-14.

14. Klein SM, Grant SA, Greengrass RA, Nielsen KC, Speer KP, White W. et al. Interscalene brachial plexus block with a continuous catheter insertion system and a disposable infusion pump. Anesth Analg 2000; 91: 1473-8.

15. Feigl G, Fuchs A, Gries M, Hogan QH, Weninger B, Rosmarin W. A supraomohyoidal plexus block designed to avoid complications. Surg Radiol Anat 2006; 28: 403-8.

16. Boezaart AP, de Beer JF, du Toit C, van Rooyen K. A new technique of continuous interscalene nerve block. Can J Anaesth 1999; 46: 275-81.

17. Oh SC, Cho HS, Ji JH, Song CH, Chung KD. The use of a continuous interscalene brachial plexus block for pain control following shoulder surgery. Korean J Anesthesiol 2007; 53: 733-9.

18. Yang CW, Jung SM, Cho CK, Kwon HU, Kang PS, Lim YS, et al. Pleural effusion and atelectasis during continuous interscalene brachial plexus block -A case report-. Korean J Anesthesiol 2010; 58 :
95-8.

19. Fredrickson MJ, Kilfoyle DH. Neurological complication analysis of 1000 ultrasound guided peripheral nerve blocks for elective orthopaedic surgery: a prospective study. Anaesthesia 2009; 64: 836-44.

20. Capdevila X, Bringuier S, Borgeat A. Infectious risk of continuous peripheral nerve blocks. Anesthesiology 2009; 110: 182-8.

21. Capdevila X, Jaber S, Pesonen P, Borgeat A, Eledjam JJ. Acute neck cellulitis and mediastinitis complicating a continuous interscalene block. Anesth Analg 2008; 107: 1419-21.

22. Morin AM, Kerwat KM, Klotz M, Niestolik R, Ruf VE, Wulf H, et al. Risk factors for bacterial catheter colonization in regional anaesthesia. BMC Anesthesiol 2005; 5: 1.

23. Capdevila X, Pirat P, Bringuier S, Gaertner E, Singelyn F, Bernard N, et al. French Study Group on Continuous Peripheral Nerve Blocks: continuous peripheral nerve blocks in hospital wards after orthopedic surgery: a multicenter prospective analysis of the quality of postoperative analgesia and complications in 1,416 patients. Anesthesiology 2005; 103: 1035-45. 